\title{
Sexualidad en el contexto de los procesos de vida de mujeres y varones con endocrinopatías: una aproximación cualitativa
}

\author{
Sexuality in the life processes context of women and men with \\ endocrinopathies: A qualitative approach
}

\author{
Loraine Ledón-Llanes,a,,*, Beatriz Torres-Rodríguez ${ }^{\mathrm{c}}$, Silvia Elena Turcios-Tristád, \\ Neida Méndez-Gómez ${ }^{\mathrm{e}}$, Marta Margarita Durand-Carbajal ${ }^{\mathrm{b}}$ \\ àepartamento de Psicología, Instituto Nacional de Endocrinología, Cuba \\ bDepartamento de Biología de la Reproducción Dr. Carlos Gual Castro, \\ Instituto Nacional de Ciencias Médicas y Nutrición Salvador Zubirán, México \\ 'Departamento de Psicología, Centro de Investigaciones Médico Quirúrgicas, Cuba \\ 'Vice-Dirección de Asistencia Médica, Instituto Nacional de Endocrinología, Cuba \\ eSociedad Cubana Multidisciplinaria de Estudios de la Sexualidad, Cuba
}

\section{Resumen}

Antecedentes: las enfermedades del sistema endocrino pueden afectar la sexualidad por sus efectos hormonales, las comorbilidades asociadas y su impacto psicosocial, tema poco estudiado desde las perspectivas de personas con estas enfermedades. Objetivos: se realizó un estudio cualitativo dirigido a comprender las experiencias sexuales de mujeres y varones con enfermedades endocrinas que producen cambios en la apariencia física. Método: se estructuró un diseño de estudio analítico-interpretativo. Participaron 68 personas entre 20 y 45 años de edad, atendidos en el Instituto Nacional de Endocrinología, La Habana, Cuba. Los instrumentos de recolección de la información utilizados fueron una planilla de datos generales, una guía de entrevista en profundidad y dos pruebas psicológicas proyectivas. Los aspectos éticos fueron considerados. Resultados: de las entrevistas en profundidad, emergieron 4 temas: 1) la expresión multidimensional de la enfermedad, 2) enfermedad y áreas de vida, 3) ejercicio de la sexualidad con la enfermedad, y 4) estrategias de afrontamiento en el área sexual; vinculados con los significados expresados sobre la salud, el cuerpo, el género y la sexualidad. Conclusiones: las/los participantes contextualizaron sus experiencias sexuales en el impacto biopsicosocial de la enfermedad. La reproducción o cuestionamiento de los significados expresados influyó en la calidad de sus experiencias sexuales. El estudio mostró que esta constituye un área de vulnerabilidad para la salud integral de las/los participantes.

Palabras clave: afrontamiento; apariencia física; enfermedades del sistema endocrino; estudio cualitativo; sexualidad; significados.

Para citar este artículo:

Ledón-Llanes, L., Torres-Rodríguez, B., Turcios-Tristá, S. E., Méndez-Gómez, N., \& Durand-Carbajal, M. M. (2020). Sexualidad en el contexto de los procesos de vida de mujeres y varones con endocrinopatías: una aproximación cualitativa. Liberabit, 26(2), e426. https://doi.org/10.24265/liberabit.2020.v26n2.06

\begin{abstract}
Background: endocrine disorders may affect sexuality due to hormonal changes, associated comorbidities and psychosocial impact. This topic remains poorly researched from the perspective of people with these conditions. Objectives: a qualitative research was conducted to understand the sexual experiences of women and men living with endocrine disorders that change their physical appearance. Methods: an analytical-interpretive study was carried out with a sample of 68 participants aged between 20 and 45, and treated at the National Institute of Endocrinology, Havana, Cuba. Data collection instruments were a personal information sheet, an in-depth interview guide and two projective psychological tests. Ethical aspects were considered. Results: in-depth interviews gave rise to four topics: 1) multidimensional expression of the disease, 2) the disease and life areas, 3) sexuality with the disease, and 4) coping strategies in the sexual area. These topics were linked to the meanings of health, body, gender and sexuality. Conclusions: the participants contextualized their sexual experiences within the biopsychosocial impact of the endocrine disorder, as well as the reproduction or questioning of the meanings that influenced the quality of their sexual experiences. The study showed that sexuality is a vulnerable area for the participants' comprehensive health.
\end{abstract}

Keywords: coping; physical appearance; endocrine disorders; qualitative research; sexuality; meanings.

Este es un artículo Open Access publicado bajo la licencia Creative Commons Atribución 4.0 Internacional. (CC-BY 4.0)

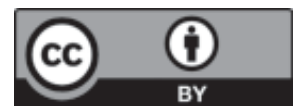

Universidad de San Martín de Porres, Lima - Perú http://ojs3.revistaliberabit.com 


\section{Introducción}

Las enfermedades endocrinas afectan el funcionamiento endocrino-metabólico de los individuos, tienen efectos sistémicos que involucran la salud sexual y reproductiva (SSR) y deterioran la calidad de vida (CV). Dichas enfermedades pueden producir disfunciones sexuales (DS) como consecuencia de sus efectos hormonales, de sus comorbilidades asociadas y de su impacto psicosocial (Balercia et al., 2007). Por lo que, en algunos casos, las DS persisten con el tratamiento y control de la enfermedad (Cintra et al., 2018; Bates et al., 2020).

El impacto psicosocial de las endocrinopatías también se relaciona con los cambios que producen en la apariencia física. Ello porque puede favorecer la construcción de significados estigmatizantes y afectar la autoimagen, el estado psíquico, las relaciones sociales y la sexualidad (Bhasin \& Basson, 2016). La percepción de atractivo físico disminuido influye en las experiencias sexuales, así como el estado neuropsicológico y psicoafectivo que también se reportan como afectados en personas con endocrinopatías (Novoa et al., 2013; Geraedts et al., 2015; O’Brien et al., 2017; Moya-Crespo, 2019).

La complejidad de esta problemática, a la que se le presta escasa atención (Kronenberg et al., 2016), requiere de una oportuna intervención y seguimiento desde una perspectiva integral y que considere las experiencias de las personas afectadas.

Considerando estos antecedentes, se realizó un estudio dirigido a comprender la experiencia de sexualidad de mujeres y varones con endocrinopatías que producen cambios en la apariencia física, en el contexto de sus procesos de vida con la enfermedad y desde sus perspectivas.

\section{Método}

Se estructuró un diseño de estudio cualitativo, analítico-interpretativo, dirigido a comprender las perspectivas de las/los participantes.

\section{Participantes}

Se incluyeron mujeres y varones adultas/os con diferentes endocrinopatías crónicas y cambios en su apariencia física debido a las mismas, atendidos en el INEN entre los años 2007 y 2017. La selección de la muestra fue por conveniencia e intencional con el propósito de obtener información amplia sobre el tema de estudio y lograr la transferibilidad de los resultados (Martínez-Salgado, 2012). Se consultaron a especialistas endocrinólogos para validar los criterios de inclusión. La muestra consistió en 68 personas entre 20 y 45 años de edad que aceptaron participar voluntariamente en el estudio previa firma del consentimiento informado. Se excluyeron personas con gonadectomía bilateral y antecedentes de radioterapia o quimioterapia.

El tamaño de la muestra total y de cada uno de los subgrupos de diagnóstico (acromegalia, hipercortisolismo endógeno [HCE], hipertiroidismo, hipotiroidismo, hiperplasia suprarrenal congénita no clásica por deficiencia de 21 hidroxilasa [HSCNCD21HO], obesidad exógena [OE]) se definió atendiendo al criterio de saturación de la información (Bonilla-García \& López-Suárez, 2016).

\section{Instrumentos}

Se utilizaron como instrumentos de recolección de la información: 1) una entrevista semiestructurada para explorar datos sociodemográficos y de salud; 2) una guía de entrevista en profundidad para obtener información subjetiva y experiencial sobre el impacto psicosocial y de salud de la enfermedad, específicamente en el área sexual y de pareja, y sobre el proceso de afrontamiento a la enfermedad y en el área sexual; y 3) dos técnicas psicológicas proyectivas, la serie de láminas somáticas (Somatic Inkblot Series [SIS]) de Cassell (1988) y el dibujo de la figura humana (DFH) de Machover (1966), para explorar percepciones somáticas e imagen corporal. 


\section{Procedimiento}

Las/los participantes fueron invitados a formar parte de este estudio de manera individual en áreas del INEN (consulta externa y hospitalización) que garantizaran la privacidad y comodidad necesarias, una vez terminada su atención de salud. El proceso de reclutamiento, consentimiento informado y aplicación de los instrumentos de recolección de la información se realizó por la misma investigadora.

Los datos sociodemográficos y de salud que permitieron caracterizar la muestra se procesaron a través de estadística descriptiva. El procesamiento de las entrevistas en profundidad tuvo carácter interpretativo, basado en la teoría fundamentada (Bonilla-García \& López-Suárez, 2016). Se realizaron lecturas repetidas de las entrevistas transcritas en detalles para localizar temas mayores, codificar la información y generar categorías (empíricas y conceptuales), las que se integraron en un esquema interpretativo a modo de propuesta de comprensión teórica del tema de estudio fundado en los datos (Bonilla-García \& López-Suárez, 2016). Las respuestas de las/los participantes a las técnicas psicológicas proyectivas se explicaron según la propuesta de interpretación de sus autores (Cassell, 1988; Machover, 1966) y complementaron el análisis de las entrevistas en profundidad. Se realizaron tres modalidades de triangulación de la información: triangulación de técnicas de recolección de la información, triangulación teórica y triangulación en investigadores (Palacios et al., 2013).

Las estrategias utilizadas para favorecer la calidad y el rigor del estudio fueron las siguientes: el relato de la historia de la investigación (definición del problema científico y aspectos metodológicos), la credibilidad (observación persistente, triangulación, comentarios de pares), la transferencia (muestreo teórico, descripción en profundidad, amplia recogida de información), los cuidados metódicos y técnicos, y la confirmación (ejercicio de la reflexión, la crítica intersubjetiva y las comparaciones) (RodríguezSabiote et al., 2005).

\section{Resultados}

\section{Características de la muestra}

En el estudio participaron 46 mujeres (67.65\%) y 22 varones (32.35\%) con diferentes diagnósticos endocrinos. Los subgrupos con mayor frecuencia fueron los de OE e hipertiroidismo (16 participantes, $23.53 \%$ cada uno), y el de menor frecuencia fue el de HSCNCD21HO (5 participantes, 7.35\%) conformado solo por mujeres porque durante el reclutamiento no se identificó a ningún varón que cumpliera los criterios de selección. Con excepción del subgrupo de acromegalia, con una distribución equitativa atendiendo al género y al diagnóstico específico (microadenoma y macroadenoma hipofisario), en el resto de los subgrupos predominaron las mujeres y diagnósticos específicos como la enfermedad de Cushing, la enfermedad de Graves-Basedow, el hipotiroidismo primario, la HSCNCD21HO y la OE grado I (ver Tabla 1).

La edad promedio fue 33.8 años (20-45 años) y aunque la distribución de la muestra fue similar entre los subgrupos de edad, las mujeres se distribuyeron con mayor frecuencia entre los 20 y 39 años (33 mujeres, $71.74 \%$ ) y los varones entre los 30 y 45 años (16 varones, 72.73\%) (ver Tabla 2).

En La Habana residían 48 participantes (70.59\%) y el resto en diferentes provincias de las zonas occidental $(11,16.18 \%)$, central $(6,8.82 \%)$ y oriental (3, 4.41\%) de Cuba. Se distribuyeron por escolaridad: 22 participantes (32.35\%) en nivel técnico-medio, 19 (27.94\%) en nivel superior, 18 (26.47\%) en nivel medio-superior y 9 (13.24\%) en nivel medio. Catorce mujeres no tenían vínculo laboral.

Refirieron tener pareja 48 participantes (70.59\%), 46 tenían vínculo «estable» y 43 convivían con la pareja. Los varones mostraron mayor tendencia a tener pareja en comparación con las mujeres (18 varones, $81.82 \%$ vs. 30 mujeres, 65.22\%) (ver Tabla 3). El tiempo medio de la relación fue de 7.9 años. Treinta y tres participantes (48.53\%) refirieron tener creencias religiosas. 
Tabla 1

Distribución de participantes según diagnóstico y género

\begin{tabular}{|c|c|c|c|c|}
\hline & JÓSTICO & Mujeres $^{1}$ & Varones $^{1}$ & Sub-total $^{2}$ \\
\hline \multirow{3}{*}{ Acromegalia } & Microadenoma hipofisario & 3 & 2 & 5 \\
\hline & Macroadenoma hipofisario & 2 & 3 & 5 \\
\hline & & $5(10.87 \%)$ & $5(22.73 \%)$ & $10(14.71 \%)$ \\
\hline \multirow{3}{*}{$\begin{array}{l}\text { Hipercortisolismo } \\
\text { endógeno }\end{array}$} & Enfermedad de Cushing & 4 & 3 & 7 \\
\hline & Síndrome de Cushing & 3 & 0 & 3 \\
\hline & & $7(15.22 \%)$ & $3(13.64 \%)$ & $10(14.71 \%)$ \\
\hline \multirow{3}{*}{ Hipertiroidismo } & Enfermedad de Graves-Basedow & 8 & 4 & 12 \\
\hline & Enfermedad nodular del tiroides & 2 & 2 & 4 \\
\hline & & $10(21.73 \%)$ & $6(27.27 \%)$ & $16(23.53 \%)$ \\
\hline \multirow{3}{*}{ Hipotiroidismo } & Hipotiroidismo primario & 6 & 2 & 7 \\
\hline & Hipotiroidismo secundario & 3 & 0 & 3 \\
\hline & & $9(19.57 \%)$ & $2(9.09 \%)$ & $11(16.18 \%)$ \\
\hline \multicolumn{2}{|c|}{$\begin{array}{l}\text { Hiperplasia suprarrenal congénita no clásica por deficiencia } \\
\text { de } 21 \text { hidroxilasa (HSCNCD21HO) }\end{array}$} & $5(10.87 \%)$ & $0(0)$ & $5(7.35 \%)$ \\
\hline \multirow{4}{*}{ Obesidad exógena } & Grado I & 5 & 2 & 7 \\
\hline & Grado II & 3 & 2 & 5 \\
\hline & Grado III (obesidad mórbida) & 2 & 2 & 4 \\
\hline & & $10(21.73 \%)$ & $6(27.27 \%)$ & $16(23.53 \%)$ \\
\hline \multicolumn{2}{|c|}{ TOTAL ${ }^{2}$} & $46(67.65 \%)$ & $22(32.35 \%)$ & $68(100 \%)$ \\
\hline
\end{tabular}

Nota: ${ }^{1}$ Los porcentajes de las columnas «mujeres» y «varones» se calcularon en función al total de mujeres (46) y varones (22), respectivamente. ${ }^{2}$ Los porcentajes de la columna Sub-total y la fila TOTAL se calcularon en función al total de muestra (68).

Tabla 2

Distribución de participantes según diagnóstico, subgrupos de edad y género

\begin{tabular}{|c|c|c|c|c|c|c|}
\hline \multirow{2}{*}{ DIAGNÓSTICO } & \multicolumn{2}{|c|}{ 20-29 años } & \multicolumn{2}{|c|}{ 30-39 años } & \multicolumn{2}{|c|}{ 40-45 años } \\
\hline & Mujeres & Varones & Mujeres & Varones & Mujeres & Varones \\
\hline Acromegalia & 1 & 2 & 0 & 2 & 4 & 1 \\
\hline Hipercortisolismo endógeno & 3 & 1 & 3 & 1 & 1 & 1 \\
\hline Hipertiroidismo & 4 & 0 & 3 & 2 & 3 & 4 \\
\hline Hipotiroidismo & 4 & 1 & 2 & 0 & 4 & 0 \\
\hline HSCNCD21HO & 2 & 0 & 3 & 0 & 0 & 0 \\
\hline Obesidad exógena & 3 & 1 & 5 & 3 & 2 & 2 \\
\hline Sub-total ${ }^{1}$ & 17 (36.96\%) & $5(22.73 \%)$ & $16(34.78 \%)$ & $8(36.36 \%)$ & $14(30.43 \%)$ & $8(36.36 \%)$ \\
\hline TOTAL $^{2}$ & \multicolumn{2}{|c|}{$22(32.35 \%)$} & \multicolumn{2}{|c|}{$24(35.29 \%)$} & \multicolumn{2}{|c|}{22 (32.35\%) } \\
\hline
\end{tabular}

Nota: ${ }^{1}$ Los porcentajes de la fila «Sub-total» se calcularon en función al total de mujeres (46) y al total de varones (22).

${ }^{2}$ Los porcentajes de la fila TOTAL se calcularon en función al total de muestra (68). 
Tabla 3

Distribución de participantes según diagnóstico, tenencia de pareja y género

\begin{tabular}{lcccc}
\hline \multirow{2}{*}{ DIAGNÓSTICO } & \multicolumn{2}{c}{ Con pareja } & \multicolumn{2}{c}{ Sin pareja } \\
\cline { 2 - 5 } & Mujeres & Varones & Mujeres & Varones \\
\hline Acromegalia & 3 & 5 & 2 & 0 \\
Hipercortisolismo endógeno & 3 & 2 & 4 & 1 \\
Hipertiroidismo & 8 & 5 & 2 & 1 \\
Hipotiroidismo & 5 & 1 & 4 & 1 \\
HSCNCD21HO & 4 & 0 & 1 & 0 \\
Obesidad exógena & 7 & 5 & 3 & 1 \\
Sub-total ${ }^{1}$ & $30(65.22 \%)$ & $18(81.82 \%)$ & $16(34.78 \%)$ & $4(18.18 \%)$ \\
\hline \multicolumn{2}{c}{ TOTAL ${ }^{2}$} & \multicolumn{2}{c}{48 (70.59\%) } & 20 (29.41\%) \\
\hline Nota: $^{1}$ Los porcentajes de la fila «Sub-total» se calcularon en función al total de mujeres (46) y al \\
total de varones (22). $^{2}$ Los porcentajes de la fila TOTAL se calcularon en función al total de muestra (68).
\end{tabular}

El tiempo promedio de convivencia con la enfermedad, considerando el tiempo promedio de diagnóstico (5.4 años) y el tiempo promedio previo de inicio de sus manifestaciones (6.6 años), fue mayor a 10 años. El subgrupo que mostró mayor tiempo promedio de diagnóstico fue el de hipotiroidismo (8.7 años); mientras que el de menor tiempo fue el de hipertiroidismo (3.3 años) (ver Tabla 4). Convivían con otras enfermedades endocrinometabólicas, sobre todo diabetes mellitus y obesidad,
35 participantes (51.47\%); y 49 (72.06\%) tenían otras comorbilidades crónicas, fundamentalmente, hipertensión arterial. Recibían atención en servicio de salud mental 2 mujeres (2.94\%), y 9 participantes (13.24\%) usaban psicofármacos. Al momento del estudio, 56 participantes (82.35\%) eran pacientes ambulatorios; 12 (17.65\%) estaban hospitalizados, fundamentalmente, mujeres con SC; y, en su mayoría, 36 participantes $(59.94 \%)$ recibían tratamiento farmacológico para la endocrinopatía (ver Tabla 4).

\section{Tabla 4}

Distribución de participantes según áreas de atención de salud, tiempo promedio desde el diagnóstico y tratamientos recibidos

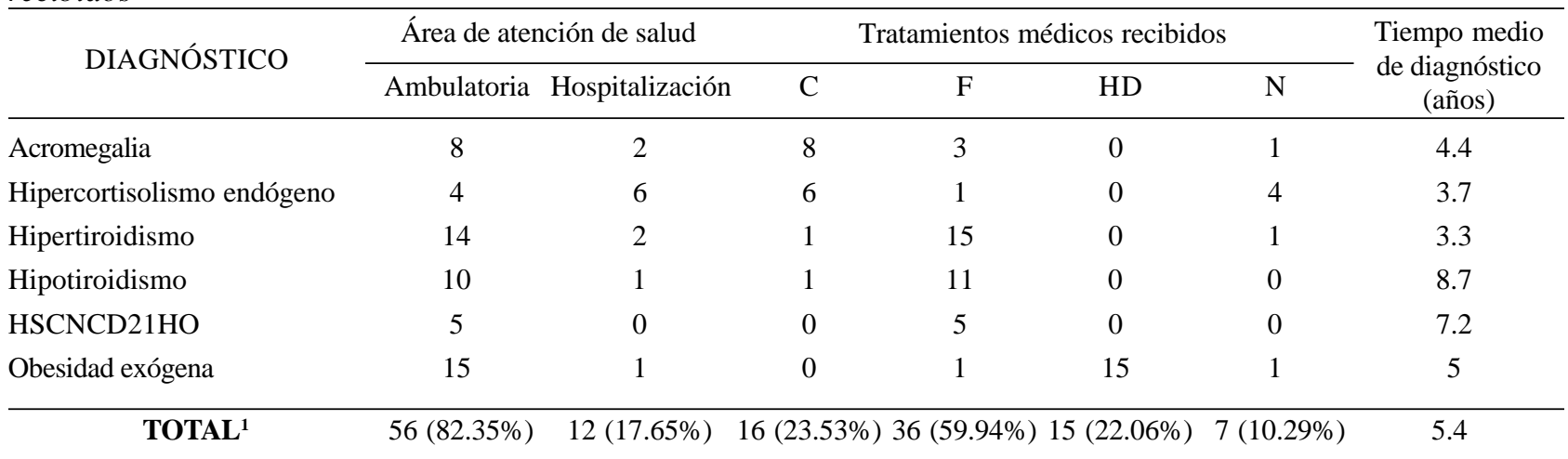

Nota: $\mathrm{C}=$ Cirugía, $\mathrm{F}=$ Farmacológico, HD = Higiénico-dietético, $\mathrm{N}=$ Ninguno. ${ }^{\mathbf{1}}$ Los porcentajes de la fila «Total» se calcularon en función al total de muestra (68). 


\section{Temas emergentes de las narrativas de las y los participantes}

Se describen los cuatro temas que emergieron de la interpretación de las narrativas de las/los participantes. Los que mostraron vínculo entre sí y contienen distintas categorías, y fueron expresados por mujeres y varones con diferentes diagnósticos, edades y que recibían atención de salud ambulatoria y en hospitalización. Se indica en el texto cuando alguna categoría se relacionó con algún grupo específico y se comparten fragmentos discursivos que fundamentaron los resultados.

\section{La expresión multidimensional de la enfermedad}

Las/los participantes refirieron que la enfermedad se expresó de forma prolongada en cuatro dimensiones de su salud: la apariencia física, los sistemas y funciones corporales, el área psicológica y la SSR.

Construyeron los cambios en su apariencia física como fuente de estigma social (como atributos asociados a devaluación social) (Goffman, 1986), como generadores de dificultades en sus vínculos sociales y como incentivadores de la búsqueda de atención a su salud. Una expresión del impacto negativo de la enfermedad sobre la apariencia física se evidenció en indicadores gráficos del DFH que se interpretan como conflictos con la imagen corporal, entre ellos: descuido en el dibujo de las figuras, refuerzo de líneas, sombreado facial o corporal, dibujo de línea media, e insuficiente diferenciación de atributos físicos femeninos y masculinos (Machover, 1966). El impacto negativo de los cambios en la apariencia física fue más destacado por las mujeres, por las/los participantes más jóvenes y por quienes tenían diagnósticos de HCE, acromegalia, HSCNCD21HO y OE. Una mujer de 23 años con $\mathrm{OE}$ (grado I) en seguimiento ambulatorio expresó malestar con su apariencia física a través del DFH realizado: «Se me parece en el cuerpo, está pasada de peso. (...) Me siento gorda, gorda, regorda, como si pesara 300 libras».

Las manifestaciones en los diferentes sistemas y funciones corporales se construyeron como limitadoras de las actividades cotidianas y generadoras de dependencias. Su frecuente presencia, como consecuencia de las endocrinopatías y las comorbilidades, agravó los malestares de salud de las/ los participantes, lo cual se reflejó en respuestas a las SIS de cuerpos y órganos enfermos (anatomía patológica) (Cassell, 1988). Así lo expresó un varón de 42 años con enfermedad de Cushing en seguimiento ambulatorio, en su respuesta a la lámina 13 de la SIS: «(¿Qué ves en la lámina?) Está con bastantes problemas... Esto pudiera ser la... la hipófisis... con problemas. (¿Qué sientes al mirar la lámina?) No me gusta, me recuerda la enfermedad... malos recuerdos.»

Respecto al área psicológica, las/los participantes refirieron haber experimentado emociones como ira, tristeza, inquietud y apatía, así como manifestaciones de agresividad, ansiedad y depresión que les generaban sentimientos de autoreproches y conductas de aislamiento social. La experiencia relatada fue de distrés psicológico (vivencia subjetiva de sufrimiento psicológico que se puede expresar en síntomas de ansiedad y depresión) (Araújo-Sol, 2012). Esta fue más destacada en participantes con acromegalia, HCE y trastornos del tiroides, y en las mujeres. Una mujer de 40 años con acromegalia (macroadenoma hipofisario) en seguimiento ambulatorio experimentó diferentes manifestaciones psicológicas antes del control de la enfermedad.

«(...) me volví una persona agresiva..., no daba golpes ni nada de eso, no era vulgar ni malas palabras ni nada, pero era demasiado exigente. Pensaba que todo lo malo iba a pasar, me preocupaba demasiado. Antes de que pasaran las cosas ya yo estaba con el tema ese dándome vueltas, discutía mucho. (...) el cerebro no paraba de pensar, no dormía (...)».

Las manifestaciones de SSR fueron consideradas como impactantes ya que afectaron a la pareja y a la familia. Además, fueron, fundamentalmente, referidas por las/los participantes más jóvenes con proyectos reproductivos y con síntomas de la 
enfermedad en esta área, como trastornos ovulatorios, espermáticos y DS. Una mujer de 31 años con HSCNCD21HO en seguimiento ambulatorio narró las dificultades vivenciadas durante su atención de salud por infertilidad.

«Ese tiempo de la asistencia a las consultas..., no me gustó, porque aquello era casi mecánico..., porque a esa hora haces todo lo que la gente te cuenta para salir embarazada: que si levanta los pies, que si ponte del otro lado. Entonces, eran una pila de técnicas que al final aplicábamos (ríe), y todo se convertía en muy maquinado cuando tiene que ser más bien, eh..., que la cosa debe correr sola. Fue un período, no sé, un poco raro».

\section{Enfermedad y áreas de vida}

Desde la perspectiva de las/los participantes, las endocrinopatías se expresaron en sus diversas dimensiones existenciales: en el ámbito individual, en las relaciones interpersonales, en las ocupaciones, en la familia y en la pareja.

En el ámbito individual, sus experiencias estuvieron mediadas por el impacto de la enfermedad en las 4 dimensiones de salud antes descritas: apariencia física, sistemas y funciones corporales, área psicológica y SSR. Dicho impacto se vivenció subjetivamente como re-construcción de sus procesos de identidad porque se autopercibían como "cambiados» en su imagen corporal, en su autovaloración y en su proyección social. Ello condujo a que se consideraran a sí mismos/ as como «diferentes» desde un sentido de devaluación, lo cual fue expresado sobre todo por las mujeres. Una mujer de 44 años con enfermedad de Graves-Basedow en seguimiento ambulatorio refirió que la enfermedad afectó integralmente a su persona: «(Respecto al impacto de la enfermedad) Mi estado emocional, mi estado sexual, mi equilibrio, mi cuerpo..., me lo hizo leña».

El impacto de la enfermedad en las relaciones interpersonales tuvo una arista positiva que se expresó en el apoyo social recibido de las amistades y de personas de la comunidad y de los espacios asistenciales de salud. Pero también tuvo una arista negativa expresada en el señalamiento de los cambios en la apariencia física y en los efectos deletéreos de los cambios psicológicos y emocionales sobre las relaciones interpersonales. Un varón de 34 años con OE (grado II) en seguimiento ambulatorio se refirió al impacto de su apariencia física en sus vínculos sociales de la siguiente manera: «Siempre me estaba comparando con las personas que yo veía (...). La gente me decía que si la gordura, la salud, boncheando, otras veces regañándome, y así (...)».

El impacto ocupacional se expresó en dificultades para ejecutar los desempeños habituales como consecuencia de las manifestaciones y secuelas de la enfermedad. Los varones expresaron mayor preocupación con la inestabilidad económica y las dependencias que les generó la enfermedad; mientras que las mujeres destacaron sus dificultades para realizar las labores domésticas y de cuidado de la familia, aun cuando tuvieran ocupaciones remuneradas. Un varón de 27 años con acromegalia (macroadenoma hipofisario), hospitalizado, narró cómo perdió su trabajo y su autonomía.

«Realmente la enfermedad no me dejó seguir en el trabajo. (...) A veces yo quiero salir, yo vivo lejos..., a buscar una medicina o algo, y no puedo hacerlo porque entonces no me da tiempo, uno se cansa mucho. (...) Y entonces uno evita eso..., y manda a otra persona, pide de favor...».

Las/los participantes refirieron que la enfermedad causó sufrimiento familiar debido a la existencia de un/a miembro de la familia enfermo/a, al impacto negativo que los cambios psicológicos y emocionales experimentados tuvieron sobre la dinámica familiar, y a la reestructuración de los roles intrafamiliares. No obstante, la familia fue considerada un apoyo fundamental en el afrontamiento a la enfermedad. Una mujer de 27 años con síndrome de Cushing, 
hospitalizada, relató cómo se afectaron sus vínculos intrafamiliares.

«iAy, mija!... la gente me decía que yo estaba loca (...). Yo no sé si estaba loca porque me alteraba, botaba a mi marido, recogía la ropa, me fajaba con mi hermano. (...) me decían, tú tienes un mal carácter. Ya después se me pasaba, jay, dios mío, que estrés! (...)».

La mayoría de las/los participantes tenía pareja y se autodefinieron como heterosexuales. Dos mujeres construyeron el vínculo de pareja desde una connotación homoerótica y bierótica e, igual al resto de las/los participantes, expresaron significados positivos respecto a esta área por sus funciones de apoyo, de favorecimiento de proyectos de vida, de vivencias emocionales positivas y como un espacio de expresión sexual.

Quienes tenían pareja a inicios de la enfermedad reconocieron el impacto negativo de los cambios psicológicos y emocionales en la relación, que en general lograron superar. Pero en algunas mujeres estas experiencias, junto al impacto de los cambios experimentados en su apariencia física, favorecieron la construcción de una imagen de sí mismas como eróticamente devaluadas, lo que desde sus perspectivas condujo al deterioro del vínculo. Una mujer de 41 años con enfermedad de GravesBasedow en seguimiento ambulatorio expuso las razones por las cuales no tenía pareja.

«Yo ahora no tengo pareja, yo quisiera tener una pareja, pero me pongo a analizar todas las cosas... Pensando en el sexo como placer y con todas las cosas que acarrea, entonces lo pongo en otro plano, ¿me entiendes? Teniendo tan poco tiempo para mi mamá y para el niño, y para mis cosas y mi salud, me digo: ¿Qué voy yo a estar buscando a nadie, me entiendes? Entonces voy desechando».

\section{Ejercicio de la sexualidad con la enfermedad}

Las/os participantes narraron diferentes experiencias sexuales con la enfermedad.

Algunas/os refirieron afectaciones sexuales que podían coexistir, tales como la disminución del deseo sexual, las dificultades con la excitación, el orgasmo y la insuficiente satisfacción sexual global. Otros malestares sexuales (expresiones de la vida sexual que, aunque pueden no constituir DS según criterios clínicos, disminuyen el bienestar sexual según la valoración subjetiva del sujeto) (Alcántara-Zavala \& Amuchástegui-Herrera, 2009) identificados fueron los siguientes: sangrado coital (mujeres), dificultades físicas o mecánicas para asumir posiciones coitales y cambios en la función eyaculatoria (varones).

Las mujeres refirieron la disminución del deseo sexual como el malestar más frecuente con la enfermedad y lo construyeron como limitador de sus motivaciones para involucrarse en la actividad coital. Esto se integró con los significados de estigma social de los cambios físicos y psíquicos experimentados y condujo a algunas mujeres a disolver su vínculo de pareja y a negarse a establecer nuevas relaciones. Una mujer de 24 años con enfermedad de Cushing, hospitalizaba, refirió desestimar las expresiones de interés sexual hacia su persona.

«(...) si miro a un muchacho o algo y..., y él me mira o algo, pienso como que él lo está haciendo pa'..., pa' mortificarme, pa' reírse de mí después o algo de eso o para cogerme pa'..., pa' su juego; que no me va a tomar en serio».

Los varones identificaron los problemas con la erección como el malestar sexual de mayor impacto, $y$, para algunos, disminuyeron sus motivaciones para la actividad sexual y generaron preocupaciones respecto a la satisfacción sexual de sus parejas. Aunque también refirieron manifestaciones psicológicas y emocionales, menor autoconfianza y 
sentido de atractivo sexual, ningún varón del estudio refirió haber disuelto su vínculo de pareja durante el proceso de la enfermedad.

Otro grupo de participantes refirió malestares sexuales similares a los antes descritos, pero los vivenciaron con menor impacto psicológico. Ello lo asociaron a la calidad de su relación de pareja, que incluía la apertura a la comunicación sobre temas sexuales y el apoyo en el afrontamiento a la enfermedad, y a que tenían información sobre los efectos sexuales de su condición de salud. Una mujer de 41 años con hipotiroidismo primario, hospitalizada, refirió que no experimentó cambios en su relación de pareja cuando vivenció dificultades sexuales.

«Disminuyó el deseo, no quería hacer nada (...). No me sentía enferma, lo que me sentía era cansada. Sí, afectó (la enfermedad) la excitación y el orgasmo también. Sabía que era algo diferente en mí, pero eso no me bajaba la autoestima ni me sentía culpable. En mi vida no permito que nada me baje la autoestima, pero tampoco lo iba a culpar a él (la pareja). Sabía que era un problema de los dos. (...) En ese sentido él me entendió, no hubo conflicto ni nada».

Un tercer grupo de participantes refirió no haber experimentado malestares sexuales a partir de la enfermedad. Aunque para ellas/os la relación de pareja también fue fundamental como fuente de apoyo, lo característico fue la expresión de una sexualidad diversa, abierta a la comunicación, a la expresión de afectos, y, en menor grado, centrada en los aspectos estéticos. Un varón de 35 años con acromegalia (microadenoma hipofisario) en seguimiento ambulatorio se refirió a la ausencia de cambios sexuales negativos.

«...todo normal, muy normal, todo muy normal. (...) perfectamente, ni cuando la operación. (...) yo creo que aumentó la frecuencia, debió aumentar la frecuencia porque la esposa mía me decía: «¿Qué fue lo que tú hiciste allá arriba?» (se refiere a la experiencia de neurocirugía). Y ahí está el resultado (embarazo de la esposa)».

\section{Estrategias de afrontamiento en el área sexual}

Las/os participantes en general utilizaron estrategias de afrontamiento a la enfermedad que combinaron recursos sociales e individuales.

En el área sexual, algunas/os aceptaron de forma acrítica sus malestares y no buscaron apoyo para mejorar su salud sexual porque desconocían el impacto de la enfermedad en esta área, y relacionaron dichos malestares con el envejecimiento o con estar viviendo una etapa de mucho distrés en sus vidas. Otras/os, aunque vincularon sus cambios sexuales con los efectos de la enfermedad, los subvaloraron debido a la severidad del impacto de la misma en otras áreas. Ello, unido a la construcción de la sexualidad como área privada, centrada en el coito y no relacionada con la salud, limitó la búsqueda de apoyo social y el disfrute de expresiones sexuales que mantenían conservadas. Significados con efectos similares se relacionaron con la autoconstrucción del sujeto en términos de pérdida de valor erótico y para la pareja, y con la disolución del vínculo de pareja en algunas mujeres, que constituyó una estrategia de afrontamiento poco efectiva porque profundizó su autodevaluación y malestar emocional.

Las estrategias de afrontamiento antes descritas fueron más utilizadas por participantes del primer grupo de experiencias sexuales. Una mujer de 38 años con síndrome de Cushing en seguimiento ambulatorio asoció su negativa a tener pareja con su profunda insatisfacción con su apariencia física.

«Los cambios como que..., me acomplejaba al..., al verme como yo estaba cambiando, como yo era antes, eso..., eso me fue afectando y me fue afectando que..., que hoy por hoy no quiero saber de ninguna relación porque yo, así como estoy de, de..., horrible... ¡mira!». 
Otras/os participantes refirieron que comunicaron sus malestares sexuales a sus parejas y encontraron la aceptación necesaria para modificar los patrones de interacción sexual y experimentar nuevas formas de disfrute erótico. Sus parejas fueron un apoyo esencial en este sentido, mediado por el manejo de significados más flexibles sobre la apariencia física y la sexualidad. En general, las/los participantes no buscaron ayuda especializada, pero quienes lo hicieron (todos varones) reportaron efectos positivos sobre su ejercicio sexual.

El uso de estrategias individuales de autovalidación, cuestionamiento de representaciones normativas sobre el cuerpo, el género y la sexualidad, y la optimización del erotismo, fue efectivo para el afrontamiento a los malestares sexuales y más referido por participantes del segundo y tercer grupos de experiencias sexuales. Un ejemplo de ello se encontró en una mujer de 35 años con OE (grado III) en seguimiento ambulatorio.

«Yo he tenido mucha suerte con los hombres porque yo he sido muy... sencilla. Yo siempre he sido sexualmente intensa (...). Yo baso mucho mi amor, mi entrega y todo eso, en el sexo. Es como mi palanca, lo que me hace moverme (...). No sé si es porque yo me siento bonita, pero yo siento que yo despierto más... sensaciones que las otras personas (...). Soy una persona que soy divertida, soy alegre. Si llego a una fiesta soy el alma de la fiesta, me tomo un trago, me pongo a bailar... es como que trato de mostrar todas mis virtudes».

\section{Integración de los resultados}

La interpretación de las narrativas de las/los participantes permitió identificar una representación de sus sexualidades en el contexto dinámico de sus procesos de vida con la enfermedad. Vivir con la endocrinopatía implicó experimentar cambios en las dimensiones corporal, psicológica y social, que modificaron sus vínculos interpersonales (incluida la pareja y la sexualidad), y movilizaron un proceso de afrontamiento dirigido a atenuar el impacto biopsicosocial de la enfermedad y favorecer la adaptación integral, incluida el área sexual.

Se identificó como vulnerabilidad para el ejercicio de la sexualidad, la reproducción de significados normativos y estereotipados sobre la apariencia física, los roles de género, la enfermedad crónica, la relación de pareja y la sexualidad. Ello se evidenció con mayor énfasis en mujeres (con diferentes diagnósticos, edades y sin pareja) para quienes la enfermedad se vivenció como devaluación erótica y utilizaron estrategias poco efectivas de afrontamiento (aislamiento social, ruptura de pareja, inhibición sexual). Otras/os mujeres y varones (con diferentes diagnósticos y edades, con o sin pareja), que refirieron significados más flexibles sobre dichas categorías, vivenciaron la enfermedad desde un sentido más conservador de su identidad y desplegaron estrategias de afrontamiento más efectivas (búsqueda de apoyo social, reestructuración del vínculo sexual y de pareja), lo que se expresó en mayor bienestar.

La integración de los temas y categorías desarrollados se muestra en la Figura 1. 


\section{Figura 1}

Integración de los resultados

\section{Sexualidad en el contexto de los procesos de vida de mujeres y varones con endocrinopatías}

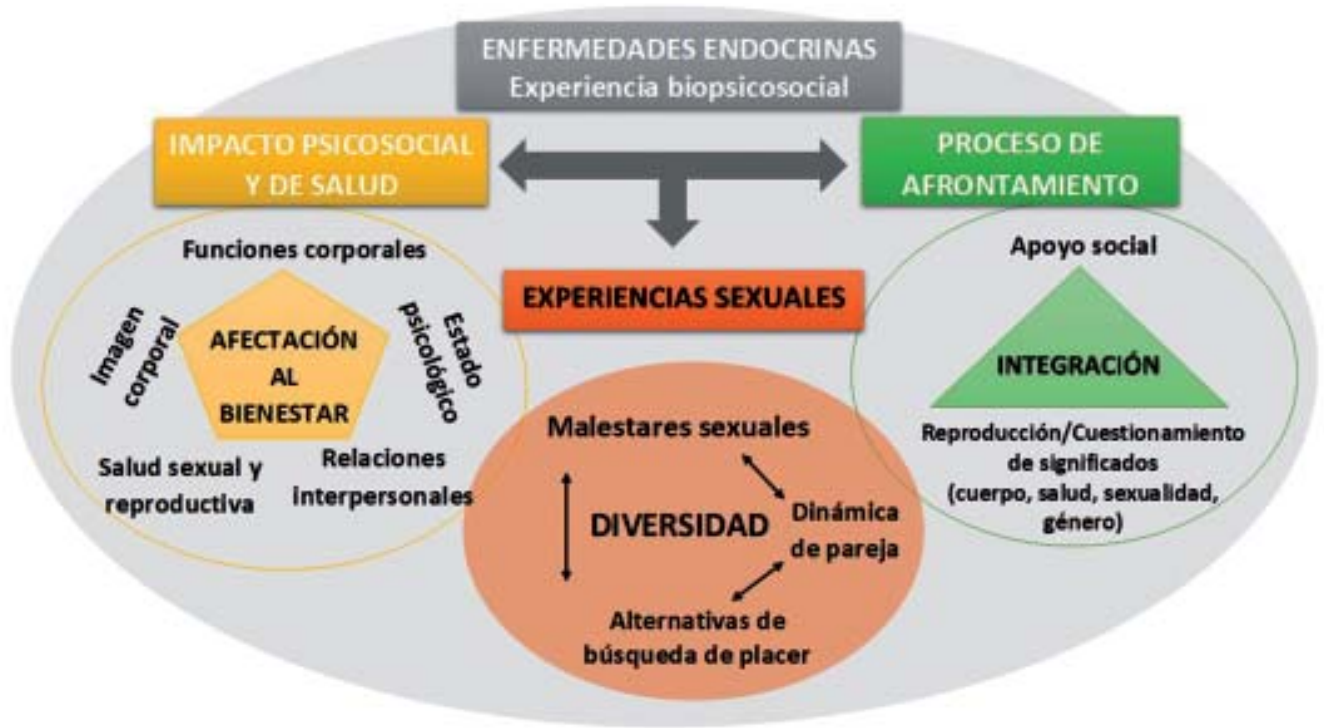

\section{Discusión}

Los resultados del estudio permiten comprender las perspectivas de personas con diferentes endocrinopatías crónicas que provocan cambios en la apariencia física acerca de sus experiencias sexuales en el contexto del impacto psicosocial y de salud de la enfermedad.

Las/os participantes definieron el impacto global de las endocrinopatías a partir de la identificación de cambios en aspectos pilares de sus identidades, lo que fundamentó la afectación a su bienestar en diferentes aristas, congruente con los efectos referidos de estas enfermedades sobre la CV (Balercia et al., 2007; Crespo et al., 2013).

La presencia de manifestaciones psicológicas profundizó el sentido de afectación al bienestar global (Brod et al., 2014), sobre todo en las mujeres; lo que puede relacionarse con su mayor riesgo de trastornos emocionales (González-Sanjuán, 2009) debido a las desigualdades y sobrecargas que favorecen los modelos patriarcales de género (Artiles et al., 2015).

La reproducción de dichos modelos también se expresó en el impacto social de la enfermedad, evidenciado en que las mayores preocupaciones de los varones se relacionaron con su rol como proveedores (Figueroa, 2014), y en las mujeres con sus roles reproductivos (González-Hernández \& Castellanos, 2014). Otra arista del impacto de la enfermedad fue el señalamiento social de los cambios en la apariencia física, como expresión de las respuestas de estigma y discriminación frente a quienes no cumplen con las normas estéticas (Pinto \& Silva, 2019).

La familia y la pareja fueron áreas valoradas como fuentes de apoyo, similar a lo referido en otras enfermedades crónicas (Soulsby \& Bennet, 2015; Grau-Ábalo, 2016). El hallazgo de mujeres con experiencias de ruptura de pareja a partir de la enfermedad expresa procesos de autodiscriminación 
asociados a estereotipos de género asimilados. Estudios sobre las relaciones de pareja en Cuba identifican la sobrevaloración de atributos físicos y la reproducción de modelos tradicionales de género (Formental-Hernández, 2015).

Coincidente con los resultados descritos por otras/ os autoras/es, las experiencias sexuales relatadas fueron diversas (Bates et al., 2020; Gabrielson et al., 2019; Martins et al., 2012), lo que está influido por diferentes aspectos psicosociales y de salud (Cintra et al., 2018; Kronenberg et al., 2016; Maiorino et al., 2018). Desde la perspectiva de las/los participantes, en dichas experiencias sobre todo influyó su estado psicológico, su sentido de atractivo sexual, la calidad del vínculo de pareja, la reproducción o cuestionamiento de significados estereotipados sobre el cuerpo, el género, y el binomio salud-enfermedad.

Dichos significados influyeron también en sus perspectivas respecto al impacto sexual de la enfermedad. Que el cambio de mayor afectación para los varones fueran las dificultades con la erección se relacionó con su significado de cuestionamiento a la identidad masculina hegemónica (González, 2010), aunque debe considerarse que es el trastorno más frecuentemente referido en esta población (Bates et al., 2020; Crawford \& Kennedy, 2016). En las mujeres, el énfasis en la disminución del deseo sexual y el impacto estético de estas enfermedades es similar a resultados obtenidos en otros estudios (Cintra et al., 2018; Krysiak et al., 2016; Pastoor et al., 2018).

Se ha identificado una tendencia a utilizar un estilo de afrontamiento pasivo, evitativo y centrado en la regulación emocional, en personas con endocrinopatías y otras enfermedades crónicas (Campbell \& Stein, 2014; Grau-Ábalo, 2016; Siegel et al., 2016). El estudio mostró que las estrategias desplegadas en el área sexual se fundamentan en los significados expresados sobre el cuerpo, el género, la sexualidad y la salud. Así, por ejemplo, la nula búsqueda de atención especializada para los malestares sexuales en las/los participantes se relacionó con la subvaloración de la sexualidad en el contexto de la enfermedad. Mientras que la ruptura de pareja utilizada por algunas mujeres se fundamentó en los modelos tradicionales de género que dictan a la mujer cumplir con normas estéticas y de disponibilidad emocional y sexual como requisito de legitimidad.

En el estudio también se identificó el uso de estrategias activas de afrontamiento, centradas en la solución de problemas (Grau-Ábalo, 2016), tales como comunicar los malestares sexuales a la pareja y buscar atención especializada (solo en algunos varones), lo que favoreció nuevas formas de disfrute erótico y expresó un proceso de afrontamiento en pareja, que es recurso esencial para el bienestar de la relación (Gabriell et al., 2016). Dichas estrategias también expresaron significados subyacentes, tales como la sexualidad como derecho humano, la relevancia del activismo sexual para la identidad masculina, y la equidad en la gestión del placer erótico en pareja.

Los hallazgos presentados permiten concluir que las personas estudiadas con endocrinopatías contextualizaron sus experiencias sexuales en el marco del impacto de la enfermedad sobre su salud integral, sobre sus procesos de identidad y sobre sus vínculos sociales. Las experiencias sexuales más asociadas con su bienestar estuvieron influidas por la calidad del vínculo de pareja, el uso de recursos sociales e individuales de afrontamiento dirigidos a favorecer la adaptación integral a la enfermedad, y el manejo de significados no normativos sobre la salud, el cuerpo, el género y la sexualidad. Estos resultados fundamentan la relevancia de considerar la sexualidad de mujeres y varones con endocrinopatías como un área de vulnerabilidad para su salud, que precisa ser valorada sistemáticamente como parte de su atención integral, y que debe abordarse desde un enfoque biopsicosocial que valide las experiencias y perspectivas de quienes con ellas conviven.

El estudio presenta algunas limitaciones, dentro de las que cabe mencionar las siguientes: la no realización de un seguimiento a las/os participantes para recabar sus experiencias sexuales en diferentes etapas con la enfermedad (por ejemplo, al momento del diagnóstico, en etapas de descontrol-control de la 
enfermedad, con posterioridad a los tratamientos médicos recibidos), la no inclusión de la perspectiva de otros actores sociales relevantes (como la pareja, la familia, las/los proveedores de la salud), y la no consideración de otras categorías psicosociales importantes para comprender el tema de estudio (por ejemplo, los procesos de personalidad, la historia psicosexual, la educación sexual, la pertenencia a grupos sociales y culturales específicos). Estas limitaciones, en lo fundamental, se relacionaron con las características de la muestra (las endocrinopatías estudiadas en general tienen muy baja incidencia, a excepción de la obesidad). Así como también con las particularidades del contexto de estudio el que, a la vez que permitió incluir a participantes con diferentes diagnósticos de endocrinopatías crónicas residentes en diversas regiones del país (por ser una institución de tercer nivel de atención de salud), impuso límites de tiempo y de espacio asociados a las características de los procesos asistenciales.

No obstante, se considera que los resultados obtenidos a través del estudio son relevantes porque han permitido iluminar un área poco estudiada e importante para la salud, la calidad de vida y el bienestar de mujeres y varones con endocrinopatías, a partir de la legitimación de sus perspectivas y experiencias. La estructuración de un marco dinámico de comprensión de algunas de las dimensiones psicosociales involucradas en las experiencias sexuales a partir de la enfermedad constituye una herramienta teórico-metodológica útil para el diseño de acciones de promoción, prevención, atención y rehabilitación de la salud sexual de estas poblaciones. Se considera también que el estudio ha permitido ratificar la utilidad metodológica del enfoque cualitativo de investigación y de sostener una perspectiva biopsicosocial en la comprensión y abordaje de las sexualidades de mujeres y varones con endocrinopatías.

\section{Conflicto de intereses}

Las autoras declaran que no existen conflicto de intereses.

\section{Responsabilidad ética}

El estudio no involucró la realización de experimentos en seres humanos ni en animales. Se han seguido los protocolos de los centros de afiliación sobre el cuidado de los participantes del estudio, el cual fue aprobado por el Consejo Científico del Instituto Nacional de Endocrinología, La Habana, Cuba (Acuerdo N 39/13), y por la Dirección de Ciencia, Tecnología e Innovación del Ministerio de Ciencia, Tecnología y Medio Ambiente, La Habana, Cuba (Aval $\mathrm{N}^{\circ}$ 1768). Se cumplieron los principios éticos de garantía de privacidad y confidencialidad de la información. La participación fue voluntaria a partir del desarrollo del proceso de consentimiento informado de forma oral y escrita.

\section{Contribución de autoría}

LLLL: concepción y diseño del estudio, recolección de datos, análisis e interpretación de los datos, elaboración, discusión y revisión final de manuscrito.

BTR: análisis e interpretación de los datos, elaboración y revisión final de manuscrito.

SETT: recolección de datos, elaboración y revisión final de manuscrito.

NMG:interpretación de los datos, elaboración y revisión final de manuscrito.

MMDC: discusión y revisión final de manuscrito.

\section{Agradecimientos}

A la Lic. Bárbara Fabré, la M. Sc. Madelín Mendoza y la M. Sc. Caridad García por su colaboración en el estudio. Al Dr. Fernando Larrea y al Dr. Juan Guillermo Figueroa por sus valiosos comentarios al artículo.

Este estudio no recibió apoyo económico por agencias financiadoras públicas, comerciales o sin fines de lucro. 


\section{Referencias}

Alcántara-Zavala, E., \& Amuchástegui-Herrera, A. (2009). Terapia sexual y normalización: significados del malestar sexual en mujeres y hombres diagnosticados con disfunción sexual. Physis Revista de Saúde Coletiva, 19(3), 591-615. https://www.scielosp.org/article/physis/ 2009.v19n3/591-615/es/

Araújo, A. L. V. L. (2012). Relações entre sintomatologia e necessidades, bem-estar e distress psicológicos [tesis de maestría, Universidad de Lisboa]. https:// repositorio.ul.pt/bitstream/10451/7648/1/ulfpie04294 5_tm.pdf

Artiles, L., Becerra-Posada, F., Faundes, A., Serruya, S. J., López, A., \& Schiavon, R. (2015). La salud de la mujer en la Región de las Américas. Revista Panamericana de Salud Pública, 37(4-5), 201-202. https:// www.scielosp.org/article/rpsp/2015.v37n4-5/201-202/es/

Balercia, G., Boscaro, M., Lombardo, F., Carosa, E., Lenzi, A., \& Jannini, E. A. (2007). Sexual Symptoms in Endocrine Diseases: Psychosomatic Perspectives. Psychoter Psychosom., 76(3), 134-140. https://doi.org/ 10.1159/000099840

Bates, J. N., Kohn, T. P., \& Pastuszak, A. W. (2020). Effect of Thyroid Hormone Derangements on Sexual Function in Men and Women. Sexual Medicine Reviews, 8(2), 217-230. https://doi.org/10.1016/j.sxmr.2018.09.005

Bhasin, Sh., \& Basson, R. (2016). Chapter 20. Sexual dysfunction in men and women. Section V. Reproduction. En Sh. Melmed, K. S. Polonsky, P. R. Larsen, \& H. R. Kronenberg, Williams Textbook of Endocrinology (13. a ed., pp. 785-830). Elsevier.

Bonilla-García, M., \& López-Suárez, A. (2016). Ejemplificación del proceso metodológico de la teoría fundamentada. Cinta de moebio, 57, 305-315. http:// dx.doi.org/10.4067/S0717-554X2016000300006

Brod, M., Pohlman, B., Hojbjerre, L., Adalsteinsson, J. E., \& Rasmussen, M. H. (2014). Impact of Adult Growth Hormone Deficiency on Daily Functioning and Wellbeing. BMC Research Notes, 7, 813. https://doi.org/ 10.1186/1756-0500-7-813

Campbell, M. M., \& Stein, D. J. (2014). Sexual Dysfunction: A Systematic Review of South African Research. South
African Medical Journal, 104(6), 439-440. http:// dx.doi.org/10.7196/SAMJ.7827

Cassell, W. A. (1988). Body Symbolism and the Somatic Inkblot Series. Northern Lights Publishing, Inc.

Cintra, M. V., Castro, M., Barreto, E. S. S., Magalhães, L. V., \& Teixeira, L. C. (2018). Impacto da acromegalia na sexualidade de homens e mulheres. Revista Psicologia, Diversidade e Saúde, 7(1), 38-46. http://dx.doi.org/ 10.17267/2317-3394rpds.v7i1.1774

Crawford, M., \& Kennedy, L. (2016). Testosterone Replacement Therapy: Role of Pituitary and Thyroid in Diagnosis and Treatment. Translational Andrology Urology, 5(6), 850-858. http://dx.doi.org/10.21037/ tau.2016.09.01

Crespo, I., Santos, A., Resmini, E., Valassi, E., MartínezMomblán, M. A., \& Webb, S. M. (2013). Improving Quality of Life in Patients with Pituitary Tumours. European Endocrinology, 9(1), 32-36. http://doi.org/ 10.17925/USE.2013.09.01.32

Figueroa, J. G. (2014). El derecho a la salud y a la vida en la experiencia de proveer económicamente. Revista Defensor, 3(8), 37-42. https://www.corteidh.or.cr/tablas/ r38850.pdf

Formental-Hernández, S. S. (2015). Servicio de Atención a Parejas, experiencia de trabajo en el Centro de Salud Mental de Playa durante el período comprendido de septiembre de 2011 a 2014. Revista Sexología y Sociedad, 21(2), 234-242. https://www.medigraphic. com/pdfs/revsexsoc/rss-2015/rss152f.pdf

Gabriel, B., Bodenmann, G., \& Beach, S. R. H. (2016). Gender Differences in Observed and Perceived Stress and Coping in Couples with a Depressed Partner. Open Journal of Depression, 5(2), 7-20. https://doi.org/ 10.4236/ojd.2016.52002

Gabrielson, A. T., Sartor, R. A., \& Hellstrom, W. J. G. (2019). The Impact of Thyroid Disease on Sexual Dysfunction in Men and Women. Sexual Medicine Reviews, 7(1), 57-70. https://doi.org/10.1016/j.sxmr.2018.05.002

Geraedts, V. J., Dimopoulou, C., Auer, M., Schopohl, J., Stalla, G. K., \& Sievers, C. (2015) Health Outcomes in Acromegaly: Depression and Anxiety are Promising Targets for Improving Reduced Quality of Life. 
Frontiers in Endocrinology, 5, 229. https://doi.org/ 10.3389/fendo.2014.00229

Goffman, E. (1986). Estigma e identidad social. En Estigma. La identidad deteriorada (pp. 11-55). Amorrortu Editores.

González-Hernández, A., \& Castellanos, B. (2014). Sexualidad y géneros desde una perspectiva humanista y crítica. En Sexualidad y géneros. Alternativas para su educación ante los retos del siglo XXI (pp. 49-146). Selvi Ediciones.

González, J. C. (2010). Masculinidad y sexualidad. En Macho, varón, masculino. Estudios de masculinidades en Cuba (pp. 63-80). Editorial de la Mujer.

González-Sanjuán, M. E. (2009). El proceso de la enfermedad desde el enfoque de género. Quaderns de ciències socials, 13, 5-54. http://roderic.uv.es/bitstream/handle/ 10550/19125/QUADERNS_13.pdf? sequence $=$ 1\&isAllowed $=\mathrm{y}$

Grau-Ábalo, J. A. (2016). Enfermedades crónicas no transmisibles: un abordaje desde los factores psicosociales. Salud \& Sociedad, 7(2), 138-166. https:/ /doi.org/10.22199/S07187475.2016.0002.00002

Kronenberg, H., Melmed, S., Larsen, P. R., \& Polonsky, K. (2016). Principles of Endocrinology. En Williams Textbook of Endocrinology (13. ${ }^{\text {a }}$ ed., pp. 1-10). Elsevier.

Krysiak, R., Drosdzol-Cop, A., Skrzypulec-Plinta, V., \& Okopien, B. (2016). Sexual Function and Depressive Symptoms in Young Women with Elevated Macroprolactin Content: A Pilot Study. Endocrine, 53, 291-298. https://doi.org/10.1007/s12020-016-0898-5

Machover, K. (1966). Dibujo de la figura humana: Un método de investigar la personalidad. En G. H. Anderson, \& H. Anderson (eds.), Técnicas Proyectivas del diagnóstico psicológico (2. ${ }^{\mathrm{a}}$ ed., pp. 393-422). Ediciones Rialp.

Maiorino, M. I., Bellastella, G., Giugliano, D., \& Esposito, K. (2018). From Inflammation to Sexual Dysfunctions: A Journey Through Diabetes, Obesity, and Metabolic Syndrome. Journal of Endocrinological Investigation, 41, 1249-1258. https://doi.org/10.1007/ s40618-018-0872-6

Martínez-Salgado, C. (2012). El muestreo en investigación cualitativa. Principios básicos y algunas controversias.
Ciencia \& Saude Coletiva, 17, 613-619. https:/www. scielosp.org/article/csc/2012.v17n3/613-619/

Martins, B., Moreira, L., Almeida, M., Toledo, T., \& Calado, J. (2012). Incidência de disfunção sexual em pacientes com obesidade e sobrepeso. Revista do Colégio Brasileiro de Cirurgiões, 40(3), 196-202. https://doi. org/10.1590/S0100-69912013000300006

Moya-Crespo, M. J. (2019). Afectación psiquiátrica y cognoscitiva en el síndrome de Cushing. Npunto, II(20), 1-25. https://www.npunto.es/content/src/pdf-articulo/ 5ddb91654b554NPvolumen20-59-83.pdf

Novoa, M. M., Vargas, R., Triana, L., \& Held, Y. (2013). Bienestar psicológico de personas con síndrome de Cushing. Diversitas, 9(1), 159-177. https://doi.org/ 10.15332/s1794-9998.2013.0001.11

O’Brien, P. O., Hinder, L. M., Callaghan, B. C., \& Feldman, E. L. (2017). Neurological Consequences of Obesity. The Lancet Neurology, 16(6), 465-477. https://doi.org/ 10.1016/S1474-4422(17)30084-4

Palacios, B., Sánchez-Gómez, M. C., \& Gutiérrez, A. (2013). Evaluar la calidad en la investigación cualitativa. Guías o checklists. En M. Vicente-Mariño, T. González- Hortigüela, \& M. Pacheco-Rueda (coord.), Actas del $2^{\circ}$ Congreso Nacional sobre Metodología de la Investigación en Comunicación (pp. 581-96). Universidad de Valladolid. https://uvadoc.uva.es/bitstream/handle/10324/3014/ EvaluarlaCalidadenlaInvestigacion.pdf?sequence $=1$

Pastoor, H., Timman, R., de Klerk, C., Bramer, W. M., Laan, E. T. M., \& Laven, J. S. E. (2018). Sexual Function in Women with Polycystic Ovary Syndrome: a Systematic Review and Meta-Analysis. Reproductive Biomedicine Online, 37(6), 750-760. https://doi.org/10.1016/ j.rbmo.2018.09.010

Pinto, F. R. M., \& Silva, C. A. B. (2019). Perfil e percepções de homens obesos mórbidos cearenses sobre a vida obesa. Revista Psicologia, Diversidade e Saúde, 8(2), 192-205. https://doi.org/10.17267/2317-3394rpds.v8i 2.2392

Rodríguez-Sabiote, C., Lorenzo-Quiles, O., \& HerreraTorres, L. (2005). Teoría y práctica del análisis de datos cualitativos. Proceso general y criterios de calidad. Revista Internacional de Ciencias Sociales y Humanidades, XV(2), 133-154. https://www.redalyc.org/ pdf/654/65415209.pdf 
Siegel, S., Milian, M., Kleist, B., Psaras, T., Tsiogka, M., Führer, D., Koltowska-Häggström, M., Honegger, J., Müller, O., Sure, U., Menzel, C., Buchfelder, M., \& Kreitschmann-Andermahr, I. (2016). Coping strategies have a strong impact on quality of life, depression, and embitterment in patients with Cushing's disease.
Pituitary, 19, 590-600. https://doi.org/10.1007/s11102016-0750-1

Soulsby, L. K., \& Bennett, K. M. (2015). Marriage and Psychological Wellbeing: The Role of Social Support. Psychology, 6(11), 1349-1359. https://doi.org/10.4236/ psych.2015.611132

Loraine Ledón-Llanes

Departamento de Psicología, Instituto Nacional de Endocrinología (INEN), Cuba; Departamento de Biología de la Reproducción Dr. Carlos Gual Castro, Instituto Nacional de Ciencias Médicas y Nutrición Salvador Zubirán (INCMNSZ), México.

Doctora en Ciencias Psicológicas e Investigadora. Su trabajo se centra en la investigación de diferentes aspectos psicosociales involucrados en los procesos de vida con enfermedades crónicas, en especial, las experiencias de género, de sexualidad y de salud sexual y reproductiva. ORCID: https://orcid.org/0000-0001-6051-030X.

Autora corresponsal: loraineaitana@gmail.com

Beatriz Torres-Rodríguez.

Departamento de Psicología, Centro de Investigaciones Médico Quirúrgicas (CIMEQ), Cuba.

Doctora en Ciencias Psicológicas, Investigadora y Profesora. Presidenta de la Sociedad Cubana Multidisciplinaria de Estudios en Sexualidad (SOCUMES). Su trabajo se centra en sexualidad de personas con enfermedades crónicas.

beatorre@infomed.sld.cu

\section{Silvia Elena Turcios-Tristá}

Vicedirección de Asistencia Médica, INEN, Cuba.

Médico Especialista de 2do. Grado de Endocrinología y Profesora. Realiza labor investigativa sobre aspectos médicos de diferentes endocrinopatías, en especial los trastornos del tiroides.

ORCID: https://orcid.org/0000-0002-4900-4542

silviaelena@infomed.sld.cu

\section{Neida Méndez-Gómez}

Sociedad Cubana Multidisciplinaria de Estudios de la Sexualidad, SOCUMES, Cuba.

Doctora en Ciencias Médicas y Especialista de 2do. Grado de Endocrinología. Realiza labor docente e investigativa en el campo de la sexología y diferentes enfermedades crónicas, en especial las endocrinopatías.

neidamendez@infomed.sld.cu

Marta Margarita Durand-Carbajal

Departamento de Biología de la Reproducción Dr. Carlos Gual Castro, Instituto Nacional de Ciencias Médicas y Nutrición Salvador Zubirán (INCMNSZ), México.

Médico Especialista e Investigadora. Realiza labor docente e investigativa en el campo de la biología de la reproducción humana. mdurand65@gmail.com 